\title{
On Quartic Fields of Signature One With Small Discriminant. II
}

\author{
By H. J. Godwin
}

Abstract. Tables of quartic fields, having two real and two complex conjugates, and with discriminants between -7776 and 0 , are given.

1. In a paper published twenty-seven years ago [1] a table of quartic fields, having two real and two complex conjugates, was given. This, before the advent of computers, had been calculated by hand and it seemed desirable to check it by computer. The opportunity has been taken to extend the table, and some features, that do not occur for very small values of the discriminant, have appeared.

One error has been discovered in [1]: the polynomial $\theta^{4}-11 \theta^{3}+43 \theta^{2}-66 \theta+$ 28 , defining the field with discriminant -1879 , has index 2 and not 1 as stated. The field with discriminant -2787 , for which the defining polynomial was given as $\theta^{4}-5 \theta^{3}+15 \theta^{2}-21 \theta+9$, with index 3 , can also be defined by the polynomial $\theta^{4}-11 \theta^{3}+38 \theta^{2}-40 \theta+3$, with index 1 .

For the sake of completeness, and with the kind permission of the Oxford Quarterly Journal of Mathematics, the original table (with the errors noted above corrected) is reprinted.

2. The method used, and established in [1], is that the field with discriminant $\Delta$, and no subfield, can be defined by a polynomial $\theta^{4}-a \theta^{3}+b \theta^{2}-c \theta+d$ with zeros $\alpha, \beta, \gamma \pm i \delta$ such that $S=(\alpha-\beta)^{2}+(\alpha-\gamma)^{2}+(\beta-\gamma)^{2}+4 \delta^{2} \leqslant$ $(-6 \Delta)^{1 / 3}$. For the present paper all polynomials with $S \leqslant 36$ have been generated by computer, thus giving all fields with $-7776 \leqslant \Delta<0$.

Since it is convenient to have defining polynomials with index 1 , and these are not necessarily given by the smallest values of $S$, some defining polynomials with larger values of $S$ are included. There may in fact be no defining polynomial with index 1 , whether or not all indices have a common factor greater than 1. (See [3, pp. 328-337], for the relevant theory.) In Table 1 below indices other than 1 are given: an asterisk indicates that the given index is a common factor of the indices of all polynomials that define the field and have $S<400$; it has not been proved that the given index is a common factor of all indices. Where there is no asterisk the indices examined have common factor 1 but no index 1 has been found.

An integral basis can usually be found by factorizing the polynomial modulo the index and discarding a repeated factor: for example, for $\Delta=-3407$, we have

Received April 4, 1983.

1980 Mathematics Subject Classification. Primary 12A30; Secondary 12A50.

Key words and phrases. Quartic fields, signature one, small discriminant. 
$\theta^{4}-9 \theta^{3}+24 \theta^{2}-34 \theta+4 \equiv \theta^{2}\left(\theta^{2}+\theta+1\right)(\bmod 2)$ and a basis is $1, \theta, \theta^{2}$, $\theta\left(\theta^{2}+\theta+1\right) / 2$. The only exceptions are referred to by special notes.

Cases where there are distinct fields with the same discriminant and no subfield can arise in two ways, either because the fields are supported (see [2]) by distinct cubic fields (as obtains for $\Delta=-4027,-7344$ ) or because they are supported by numbers from the same cubic field whose ratio is not a square (as obtains for $\Delta=-3776,-6571,-6848,-6883,-6928)$.

Quartic fields with subfields are of the form $K(\sqrt{\mu})$ : those with discriminants in the relevant interval are given in Table 2.

TABLE 1

\begin{tabular}{|c|c|c|c|c|c|c|c|c|c|c|c|}
\hline & $a$ & $b$ & $c$ & $d$ & Index & & $a$ & $b$ & $c$ & $d$ & index \\
\hline-283 & 3 & 7 & 5 & 1 & & -2319 & 9 & 28 & 33 & 12 & \\
\hline-331 & 5 & 10 & 6 & 1 & & -2327 & 8 & 23 & 27 & 8 & \\
\hline-491 & 7 & 15 & 9 & 1 & & -2412 & 9 & 29 & 39 & 16 & \\
\hline-563 & 6 & 12 & 7 & 1 & & -2443 & 8 & 24 & 29 & 9 & \\
\hline-643 & 7 & 18 & 18 & 5 & & -2480 & 8 & 24 & 30 & 10 & \\
\hline-688 & 4 & 10 & 6 & 1 & & -2488 & 9 & 31 & 46 & 22 & \\
\hline-731 & 5 & 11 & 11 & 3 & & -2563 & 6 & 16 & 19 & 7 & \\
\hline-751 & 6 & 13 & 11 & 1 & & -2608 & 8 & 22 & 22 & 2 & \\
\hline-848 & 8 & 23 & 26 & 9 & & -2619 & 9 & 27 & 29 & 9 & \\
\hline-976 & 6 & 15 & 16 & 5 & & -2687 & 11 & 42 & 59 & 14 & \\
\hline-1099 & 7 & 19 & 21 & 7 & & -2696 & 7 & 18 & 17 & 3 & \\
\hline-1107 & 7 & 18 & 18 & 3 & & -2736 & 6 & 14 & 12 & 1 & \\
\hline-1192 & 5 & 11 & 10 & 2 & & -2763 & 5 & 11 & 7 & 1 & \\
\hline-1255 & 7 & 15 & 8 & 1 & & -2764 & 7 & 17 & 11 & 2 & \\
\hline-1328 & 4 & 9 & 6 & 1 & & -2767 & 7 & 18 & 17 & 4 & \\
\hline-1371 & 4 & 8 & 7 & 1 & & -2787 & 11 & 38 & 40 & 3 & \\
\hline-1399 & 7 & 16 & 11 & 2 & & -2816 & 12 & 50 & 80 & 31 & \\
\hline-1423 & 7 & 19 & 22 & 7 & & -2824 & 7 & 17 & 14 & 2 & \\
\hline-1424 & 8 & 25 & 34 & 15 & & -2843 & 5 & 11 & 9 & 1 & \\
\hline-1456 & 8 & 22 & 22 & 5 & & -2859 & 9 & 29 & 37 & 11 & \\
\hline-1472 & 6 & 14 & 14 & 3 & & -2911 & 8 & 25 & 35 & 16 & \\
\hline-1588 & 3 & 9 & 8 & 2 & & -2943 & 7 & 18 & 19 & 4 & \\
\hline-1732 & 9 & 27 & 28 & 2 & & -3052 & 7 & 19 & 23 & 8 & \\
\hline-1791 & 7 & 17 & 14 & 1 & & -3119 & 3 & 9 & 6 & 1 & \\
\hline-1823 & 9 & 30 & 41 & 16 & & -3163 & 10 & 34 & 41 & 7 & \\
\hline-1856 & 10 & 35 & 46 & 11 & & -3175 & 8 & 23 & 25 & 8 & \\
\hline-1879 & 11 & 43 & 66 & 28 & $2^{*}$ & -3188 & 7 & 19 & 20 & 6 & \\
\hline-1927 & 9 & 28 & 31 & 4 & & -3216 & 10 & 35 & 48 & 21 & \\
\hline-1931 & 8 & 24 & 29 & 11 & & -3223 & 9 & 29 & 36 & 11 & \\
\hline-1963 & 7 & 20 & 26 & 11 & & -3267 & 5 & 12 & 12 & 3 & \\
\hline-1968 & 6 & 14 & 10 & 2 & & -3271 & 4 & 9 & 9 & 2 & \\
\hline-1984 & 6 & 14 & 12 & 2 & & -3284 & 7 & 17 & 16 & 2 & \\
\hline-2051 & 9 & 29 & 37 & 15 & & -3303 & 8 & 23 & 25 & 7 & \\
\hline-2068 & 9 & 28 & 33 & 11 & & -3376 & 6 & 13 & 8 & 1 & \\
\hline-2092 & 9 & 27 & 29 & 8 & & -3407 & 9 & 29 & 34 & 4 & $2^{*}$ \\
\hline-2096 & 8 & 22 & 22 & 6 & & -3411 & 3 & 8 & 6 & 1 & \\
\hline-2116 & 5 & 10 & 9 & 1 & & -3424 & 8 & 21 & 18 & 2 & \\
\hline-2151 & 8 & 23 & 25 & 4 & & -3431 & 9 & 29 & 38 & 12 & $2^{*}$ \\
\hline-2183 & 6 & 15 & 15 & 4 & & -3436 & 9 & 28 & 34 & 10 & \\
\hline-2191 & 5 & 12 & 13 & 4 & & -3504 & 6 & 16 & 18 & 6 & \\
\hline-2219 & 5 & 12 & 14 & 5 & & -3544 & 11 & 41 & 58 & 26 & \\
\hline-2243 & 9 & 28 & 32 & 5 & & -3559 & 8 & 19 & 11 & 1 & \\
\hline-2284 & 9 & 26 & 24 & 2 & & -3571 & 5 & 13 & 7 & 1 & \\
\hline
\end{tabular}


TABLE 1 (continued)

\begin{tabular}{|c|c|c|c|c|c|c|c|c|c|c|c|}
\hline & $a$ & $b$ & $c$ & $d$ & Index & & $a$ & $b$ & $c$ & $d$ & Index \\
\hline-3632 & 10 & 36 & 52 & 23 & & -5260 & 9 & 31 & 45 & 20 & \\
\hline-3723 & 9 & 31 & 45 & 21 & & -5323 & 10 & 36 & 53 & 22 & \\
\hline-3747 & 7 & 19 & 21 & 5 & & -5343 & 10 & 35 & 49 & 19 & \\
\hline-3751 & 4 & 13 & 7 & 1 & & -5348 & 5 & 13 & 16 & 6 & \\
\hline-3776 & 2 & 9 & 6 & 1 & & -5371 & 10 & 34 & 43 & 15 & \\
\hline-3776 & 10 & 32 & 34 & 9 & & -5424 & 6 & 14 & 14 & 2 & \\
\hline-3816 & 11 & 44 & 73 & 37 & & -5431 & 8 & 19 & 9 & 1 & \\
\hline-3888 & 12 & 48 & 70 & 27 & & -5432 & 9 & 30 & 43 & 19 & \\
\hline-3891 & 5 & 14 & 12 & 3 & & -5448 & 13 & 59 & 106 & 58 & \\
\hline-3899 & 6 & 14 & 13 & 1 & & -5476 & 9 & 29 & 36 & 14 & \\
\hline-3919 & 8 & 25 & 33 & 13 & & -5548 & 9 & 30 & 40 & 14 & \\
\hline-3951 & 5 & 11 & 10 & 1 & & -5552 & 12 & 50 & 82 & 42 & \\
\hline-3967 & 7 & 19 & 18 & 4 & $2^{*}$ & -5568 & 10 & 34 & 42 & 11 & \\
\hline-3984 & 6 & 19 & 24 & 9 & 3 & -5591 & 11 & 38 & 41 & 9 & $2^{*}$ \\
\hline-4027 & 7 & 16 & 8 & 1 & & -5595 & 5 & 13 & 15 & 5 & \\
\hline-4027 & 8 & 22 & 23 & 4 & & -5636 & 7 & 23 & 36 & 18 & 3 \\
\hline-4063 & 11 & 42 & 61 & 22 & & -5644 & 11 & 43 & 65 & 22 & \\
\hline-4103 & 7 & 19 & 14 & 3 & & -5675 & 10 & 34 & 43 & 17 & \\
\hline-4108 & 7 & 18 & 18 & 2 & & -5732 & 5 & 15 & 20 & 8 & 2 \\
\hline-4152 & 8 & 23 & 26 & 4 & 2 & -5748 & 11 & 37 & 36 & 8 & 2 \\
\hline-4192 & 8 & 25 & 34 & 14 & & -5755 & 9 & 30 & 40 & 17 & \\
\hline-4204 & 3 & 11 & 9 & 2 & & -5792 & 12 & 51 & 88 & 46 & \\
\hline-4287 & 7 & 20 & 27 & 12 & & -5816 & 9 & 28 & 31 & 5 & \\
\hline-4319 & 4 & 11 & 9 & 2 & & -5867 & 6 & 14 & 11 & 1 & \\
\hline-4384 & 12 & 47 & 60 & 2 & & -5932 & 11 & 43 & 69 & 34 & \\
\hline-4423 & 9 & 27 & 28 & 5 & & -5963 & 9 & 27 & 25 & 7 & \\
\hline-4432 & 10 & 43 & 44 & 13 & & -5987 & 6 & 16 & 17 & 5 & \\
\hline-4491 & 8 & 22 & 21 & 3 & & -6043 & 7 & 21 & 27 & 11 & \\
\hline-4492 & 5 & 14 & 10 & 2 & & -6064 & 10 & 37 & 56 & 25 & \\
\hline-4503 & 6 & 17 & 11 & 2 & & -6071 & 5 & 12 & 11 & 2 & \\
\hline-4564 & 9 & 26 & 21 & 5 & & -6075 & 9 & 30 & 40 & 15 & \\
\hline-4568 & 7 & 20 & 25 & 9 & & -6079 & 12 & 51 & 85 & 38 & \\
\hline-4595 & 10 & 36 & 51 & 19 & & -6091 & 8 & 26 & 37 & 17 & \\
\hline-4615 & 7 & 19 & 20 & 5 & & -6124 & 9 & 24 & 16 & 2 & \\
\hline-4648 & 5 & 12 & 7 & 1 & & -6199 & 7 & 19 & 22 & 4 & $2^{*}$ \\
\hline-4652 & 8 & 24 & 26 & 8 & 2 & -6283 & 12 & 52 & 91 & 46 & \\
\hline-4663 & 7 & 20 & 23 & 8 & & -6331 & 7 & 21 & 29 & 13 & \\
\hline-4671 & 6 & 15 & 15 & 3 & & -6343 & 17 & 89 & 142 & 68 & $2^{*}$ \\
\hline-4675 & 13 & 60 & 110 & 55 & & -6371 & 9 & 28 & 32 & 11 & \\
\hline-4703 & 12 & 51 & 87 & 46 & & -6387 & 9 & 26 & 24 & 3 & \\
\hline-4744 & 7 & 21 & 24 & 8 & 2 & -6399 & 8 & 21 & 15 & 3 & \\
\hline-4748 & 11 & 42 & 62 & 26 & & -6411 & 12 & 52 & 93 & 51 & \\
\hline-4752 & 8 & 21 & 18 & 3 & & -6444 & 7 & 20 & 26 & 10 & \\
\hline-4780 & 6 & 18 & 22 & 8 & 2 & -6480 & 16 & 87 & 178 & 97 & 3 \\
\hline-4799 & 9 & 26 & 23 & 1 & $2^{*}$ & -6484 & 11 & 41 & 52 & 2 & \\
\hline-4832 & 8 & 23 & 24 & 2 & & -6507 & 10 & 30 & 23 & 5 & \\
\hline-4864 & 8 & 24 & 28 & 10 & & -6571 & 4 & 10 & 7 & 1 & \\
\hline-4907 & 11 & 43 & 65 & 23 & & -6571 & 8 & 22 & 21 & 4 & \\
\hline-4944 & 8 & 23 & 24 & 3 & & -6571 & 9 & 28 & 32 & 9 & \\
\hline-4979 & 11 & 44 & 72 & 37 & & -6603 & 8 & 22 & 21 & 5 & \\
\hline-4999 & 10 & 35 & 47 & 17 & & -6604 & 11 & 43 & 67 & 32 & \\
\hline-5036 & 11 & 44 & 72 & 34 & & -6611 & 9 & 30 & 40 & 13 & \\
\hline-5184 & 6 & 15 & 14 & 3 & & -6656 & 8 & 24 & 28 & 9 & \\
\hline-5224 & 11 & 39 & 46 & 10 & & -6664 & 11 & 42 & 61 & 19 & \\
\hline-5231 & 9 & 26 & 23 & 6 & & -6687 & 9 & 29 & 36 & 13 & \\
\hline-5243 & 9 & 24 & 16 & 1 & & -6691 & 3 & 7 & 7 & 1 & \\
\hline
\end{tabular}




\begin{tabular}{|c|c|c|c|c|c|c|c|c|c|c|c|}
\hline & $a$ & $b$ & $c$ & $d$ & Index & & $a$ & $b$ & $c$ & $d$ & Index \\
\hline-6700 & 9 & 25 & 19 & 4 & & -7199 & 7 & 18 & 9 & 1 & $2^{*}$ \\
\hline-6739 & 11 & 24 & 20 & 3 & & -7259 & 4 & 10 & 11 & 3 & \\
\hline-6763 & 11 & 39 & 47 & 15 & & -7331 & 7 & 18 & 14 & 3 & \\
\hline-6791 & 11 & 44 & 71 & 32 & & -7335 & 10 & 35 & 47 & 13 & \\
\hline-6800 & 8 & 23 & 26 & 5 & & -7344 & 10 & 36 & 52 & 19 & \\
\hline-6848 & 2 & 8 & 6 & 1 & & -7344 & 8 & 21 & 14 & 1 & 3 \\
\hline-6848 & 6 & 17 & 22 & 9 & & -7351 & 10 & 39 & 62 & 28 & $4^{* \dagger \dagger}$ \\
\hline-6863 & 11 & 41 & 54 & 7 & & -7407 & 8 & 25 & 33 & 12 & \\
\hline-6880 & 12 & 49 & 76 & 28 & $2^{+}$ & -7412 & 5 & 12 & 13 & 3 & \\
\hline-6883 & 6 & 16 & 13 & 3 & & -7463 & 15 & 71 & 110 & 52 & $2^{*}$ \\
\hline-6883 & 7 & 16 & 10 & 1 & & -7472 & 8 & 26 & 38 & 18 & \\
\hline-6883 & 10 & 34 & 43 & 16 & & -7492 & 7 & 20 & 26 & 8 & $2^{*}$ \\
\hline-6896 & 12 & 47 & 64 & 21 & & -7528 & 5 & 13 & 10 & 2 & \\
\hline-6912 & 12 & 48 & 68 & 18 & & -7532 & 7 & 20 & 12 & 2 & \\
\hline-6924 & 9 & 29 & 35 & 8 & & --7571 & 7 & 19 & 19 & 5 & \\
\hline-6928 & 8 & 25 & 24 & 7 & & -7652 & 5 & 16 & 13 & 3 & \\
\hline-6928 & 8 & 19 & 10 & 1 & & -7668 & 9 & 27 & 28 & 6 & \\
\hline-6939 & 8 & 24 & 31 & 11 & & -7692 & 25 & 211 & 627 & 218 & \\
\hline-6967 & 9 & 30 & 43 & 17 & $2^{*}$ & -7699 & 7 & 18 & 16 & 3 & \\
\hline-6976 & 10 & 37 & 56 & 26 & & -7703 & 8 & 23 & 27 & 7 & \\
\hline-6987 & 41 & 560 & 2598 & 723 & & -7715 & 10 & 32 & 33 & 5 & \\
\hline-7087 & 6 & 17 & 21 & 8 & & -7732 & 11 & 44 & 70 & 32 & $2^{*}$ \\
\hline-7088 & 8 & 20 & 14 & 2 & & -7744 & 10 & 33 & 36 & 2 & \\
\hline-7155 & 7 & 18 & 20 & 5 & & -7771 & 10 & 36 & 51 & 20 & \\
\hline
\end{tabular}

${ }^{\dagger}$ A basis for the field is $1, \theta, \theta^{2}, \theta^{2}(\theta-1) / 2$ but not $1, \theta, \theta^{2}, \theta\left(\theta^{2}-1\right) / 2$.

${ }^{\dagger \dagger}$ Polynomials defining this field were examined for $S<1000$. No index 2 occurs, but 2 is the highest common factor of all indices examined. A basis for the field is $1, \theta, \theta(\theta-1) / 2, \theta^{2}(\theta-1) / 2$.

TABLE 2

\begin{tabular}{cl}
$\Delta$ & \multicolumn{1}{c}{$\mu$} \\
-275 & $-(1+3 \sqrt{5}) / 2$ \\
-400 & $(1+\sqrt{5}) / 2$ \\
-448 & $-1+2 \sqrt{2}$ \\
-475 & $-1+2 \sqrt{5}$ \\
-507 & $(-1+\sqrt{13}) / 2$ \\
-775 & $(-1+5 \sqrt{5}) / 2$ \\
-1024 & $11+\sqrt{2}$ \\
-1156 & $14+\sqrt{17}$ \\
-1323 & $(3+\sqrt{21}) / 2$ \\
-1375 & $15+4 \sqrt{5}$ \\
-1472 & $-3+4 \sqrt{2}$ \\
-1475 & $(3+7 \sqrt{5}) / 2$ \\
-1600 & $11+\sqrt{5}$ \\
-1728 & $13+2 \sqrt{3}$ \\
-1775 & $-3+4 \sqrt{5}$ \\
-1792 & $11+2 \sqrt{2}$ \\
-1975 & $(-17+11 \sqrt{5}) / 2$
\end{tabular}

\begin{tabular}{cl}
$\Delta$ & \multicolumn{1}{c}{$\mu$} \\
-1984 & $11+4 \sqrt{2}$ \\
-2000 & $1 \sqrt{5}$ \\
-2048 & $1 \sqrt{2}$ \\
-2312 & $(3+\sqrt{17}) / 2$ \\
-2375 & $(-5+9 \sqrt{5}) / 2$ \\
-2475 & $(3+9 \sqrt{5}) / 2$ \\
-2704 & $(3+\sqrt{13}) / 2$ \\
-3008 & $-5+6 \sqrt{2}$ \\
-3275 & $(-9+11 \sqrt{5}) / 2$ \\
-3312 & $15+4 \sqrt{3}$ \\
-3312 & $-5+4 \sqrt{3}$ \\
-3475 & $1(7+11 \sqrt{5}) / 2$ \\
-3600 & $(3+3 \sqrt{5}) / 2$ \\
-3775 & $(-1+11 \sqrt{5}) / 2$ \\
-3875 & $-5+6 \sqrt{5}$ \\
-3887 & $(-5+3 \sqrt{13}) / 2$ \\
-4032 & $13+6 \sqrt{2}$
\end{tabular}


TABLE 2 (continued)

\begin{tabular}{cl}
\multicolumn{1}{c}{$\Delta$} & \multicolumn{1}{c}{$\mu$} \\
-4107 & $(-5+\sqrt{37}) / 2$ \\
-4275 & $13+6 \sqrt{5}$ \\
-4400 & $(1+3 \sqrt{5}) / 2$ \\
-4475 & $-1+6 \sqrt{5}$ \\
-4544 & $-1+6 \sqrt{2}$ \\
-4608 & $11+\sqrt{3}$ \\
-4608 & $-1+\sqrt{3}$ \\
-4775 & $(-9+13 \sqrt{5}) / 2$ \\
-4975 & $(7+13 \sqrt{5}) / 2$ \\
-5056 & $-7+8 \sqrt{2}$ \\
-5275 & $(-1+13 \sqrt{5}) / 2$ \\
-5488 & $121+8 \sqrt{7}$ \\
-5491 & $17+2 \sqrt{17}$ \\
-5616 & $13+4 \sqrt{3}$ \\
-5616 & $-3+4 \sqrt{3}$ \\
-5887 & $(-1+\sqrt{29}) / 2$ \\
-5888 & $13+4 \sqrt{2}$ \\
-5975 & $(-13+15 \sqrt{5}) / 2$
\end{tabular}

\begin{tabular}{cl}
\multicolumn{1}{c}{$\Delta$} & \multicolumn{1}{c}{$\mu$} \\
-6275 & $(11+15 \sqrt{5}) / 2$ \\
-6336 & $11+2 \sqrt{3}$ \\
-6336 & $-1+2 \sqrt{3}$ \\
-6591 & $(-13+5 \sqrt{13}) / 2$ \\
-6592 & $15+8 \sqrt{2}$ \\
-6724 & $132+5 \sqrt{41}$ \\
-6768 & $11+4 \sqrt{3}$ \\
-6768 & $-1+4 \sqrt{3}$ \\
-6775 & $-7+8 \sqrt{5}$ \\
-6912 & $1 \sqrt{3}$ \\
-6975 & $(3+15 \sqrt{5}) / 2$ \\
-7267 & $13+2 \sqrt{13}$ \\
-7375 & $15+8 \sqrt{5}$ \\
-7600 & $11+2 \sqrt{5}$ \\
-7616 & $-3+8 \sqrt{2}$ \\
-7616 & $-9+10 \sqrt{2}$ \\
-7775 & $-3+8 \sqrt{5}$
\end{tabular}

Department of Statistics and Computer Science

Royal Holloway College

Egham Hill

Egham

Surrey TW20 0EX, England

1. H. J. GoDwin, "On quartic fields of signature one with small discriminant," Quart. J. Math. Oxford Ser. (2), v. 8, 1957, pp. 214-222.

2. H. J. Godwin, "On relations between cubic and quartic fields," Quart. J. Math. Oxford Ser. (2), v. 13, 1962, pp. 206-212.

3. H. HASSE, Zahlentheorie, Akademie-Verlag, Berlin, 1949. 